\title{
Multi-mJ terahertz generation in periodically poled Lithium-Niobate by pulse recycling with dispersion compensation
}

\author{
Lu Wang ${ }^{1,2, *}$, Arya Fallahi $^{1}$, Koustuban Ravi ${ }^{1,3}$ and Franz Kärtner ${ }^{1,2,4}$ \\ ${ }^{1}$ Center for Free Electron Laser Science, Deutsches Elektronen-Synchrotron Notkestra ße 85, 22607 \\ ${ }^{2}$ Department of Physics, Universität of Hamburg, Luruper Chaussee 149, 22761 Hamburg, Germany \\ ${ }^{3}$ Research Laboratory of Electronics, Massachusetts Institute of Technology (MIT), Cambridge, \\ Massachusetts 02139, USA \\ ${ }^{4}$ The Hamburg Centre for Ultrafast Imaging (CUI), Luruper Chaussee 149, 22761 Hamburg, \\ Germany
}

\begin{abstract}
We propose a design for high efficiency narrow-band terahertz generation using multiple stages with dispersion compensation. Terahertz efficiencies of a few percent are predicted.
\end{abstract}

\section{Introduction}

High energy multi-cycle (narrow band) terahertz pulses can be utilized for linear electron acceleration [1]. This opens up the possibilities for developing table-top X-ray sources [2]. Due to the finite damage threshold of periodically poled Lithium Niobate (PPLN) crystals, absorption of the material at terahertz frequencies and low quantum efficiency, generation of terahertz radiation with high energy is extremely challenging.

Our simulations suggest that with a pulse-train format input pump pulse [3], the efficiency of a single PPLN stage $\eta=1.04 \%$ can be obtained at the optimal pump pulse duration $\tau_{\text {FWHM }} 150 \mathrm{ps}$ with an input fluence of $0.83 \mathrm{~J} / \mathrm{cm}^{2}$ and terahertz absorption $\alpha(0.3$ $\mathrm{THz})=1.3 / \mathrm{cm}(80 \mathrm{~K})[4]$.

Here, we consider a consecutive arrangement of PPLN stages (see Fig. 1.), which recycles the pump pulse for further terahertz generation. The arrangement increases the effective length ( $\mathrm{L}_{\text {eff }}$ ) and circumvents excessive terahertz absorption through the outcoupling of the terahertz pulse after each stage.

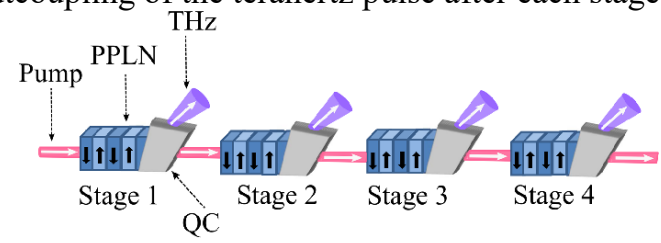

Fig. 1. Schematic illustration of the multi-stage system. QC represents the quartz coupler.

\footnotetext{
${ }^{*}$ Corresponding author: $\underline{\text { lu.wang } @ \text {,desy.de }}$
} 


\section{Results}

\subsection{Pulse recycling with dispersion compensation}

For a 4-stage system, terahertz spectra and the corresponding efficiencies are shown in Fig. 2(a) and Fig. 2(c), respectively. One can see that the terahertz conversion efficiency reduces subsequently after each stage. Due to the dispersion, different frequency elements in the pump spectrum pick up different phases. Consequently, the terahertz radiation generated from different spectral ranges of the pump pulse carries different phases, leading to partial destructive interference. This leads to the reduction in efficiency. In order to mitigate the efficiency reduction due to the dispersion effect, we compensate the Lithium Niobate (LN) dispersion of the pump pulse accumulated by propagation before the pump pulse is recycled to the next PPLN stage. One can see that the dispersion compensation significantly enhances the terahertz conversion efficiency (see Fig.2(b,d)).
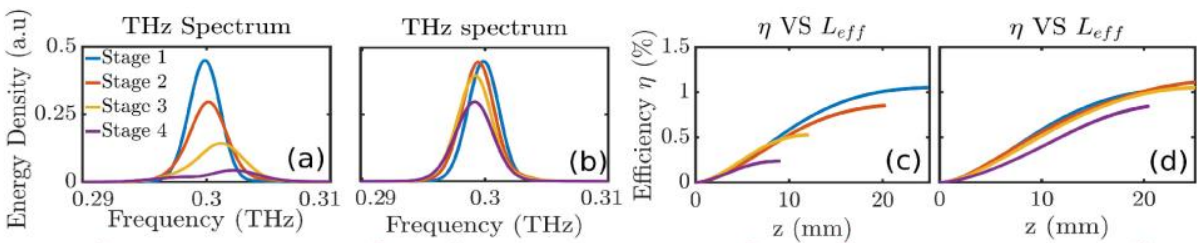

Fig. 2. (a,c) show the terahertz spectrum and the efficiency of a 4-stage system by direct pump recycling. $(b, d)$ show the terahertz spectrum and the efficiency of a 4-stage system with dispersion compensation.

\subsection{Pump pulse spectral dynamics}

In order to further analyse the dispersion effect on the terahertz generation process, we perform the short time Fourier transform (STFT) of the output pump pulse after a single PPLN stage. The STFT reflects the pump pulse spectral distribution with respect to time (i.g instantaneous spectrum). As shown in Fig. 3(b), the pump pulse spectrum versus time forms a ' $U$ ' shape due to the cascading effect. The maximum cascading occurs where the maximum intensity is located and thus causes maximum spectral down shift forming the ' $U$ ' shape. This drastic spectral variation in the time domain reduces terahertz generation.
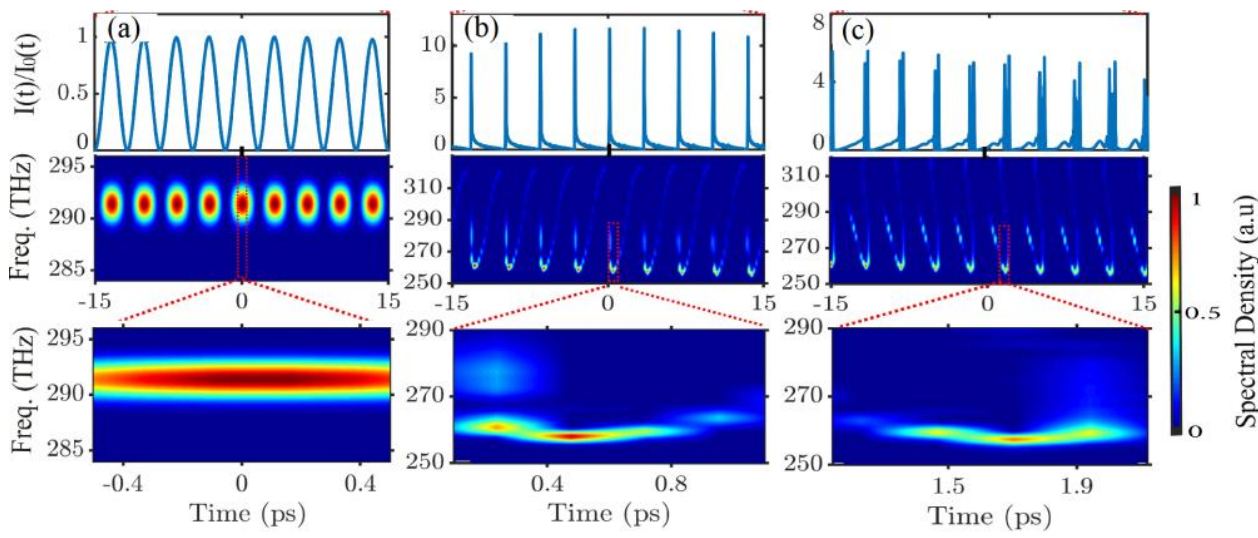
Fig. 3. (a) represents the temporal profile of the input pump pulse. (b) represents the temporal profile of the output pump pulse after a $25 \mathrm{~mm}$ PPLN crystal (c) represents the temporal profile of (b) after dispersion compensation. The coloured 2-D plots correspond to STFT.

Compensating for the dispersion during propagation through the crystal in each stage smooths the ' $U$ ' shape as shown in STFT in Fig.3(c). The spectral variation in time is reduced, and thus the terahertz generated locally in time can add up coherently leading to higher efficiency.

\section{Conclusions}

We show that a pump pulse recycling scheme can increase the terahertz generation efficiency. Additionally, by implementing dispersion compensation, the generation efficiency can be further enhanced.

\section{References}

1. E. A. Nanni, W. R. Huang, K.-H. Hong, K. Ravi, A. Fallahi, G. Moriena, R. D. Miller, and F. X. Kärtner, "Terahertz-driven linear electron acceleration," Nat. Commu, vol. 6, p. 8486, (2015).

2. F. Kärtner, F. Ahr, A.-L. Calendron, H. Çankaya, S. Carbajo, G. Chang, G. Cirmi, K. Dörner, U. Dorda, A. Fallahi, et al., "Axsis: Exploring the frontiers in attosecond x-ray science, imaging and spectroscopy," Nuclear Instruments and Methods in Physics Research Section A: Accelerators, Spectrometers, Detectors and Associated Equipment, (2016).

3. K. Ravi, D. N. Schimpf, and F. X. Kärtner, "Pulse sequences for efficient multi-cycle terahertz generation in periodically poled lithium niobate," Opt. Express. 24, no. 22, pp. 25582-25607, (2016).

4. X. Wu, C. Zhou, W. R. Huang, F. Ahr, and F. X. Kärtner, "Temperature dependent refractive index and absorption coefficient of congruent lithium niobate crystals in the terahertz range," Opt. Express 23, no. 23, pp. 29729-29737, (2015). 